Fetal Diagnosis and Therapy
Fetal Diagn Ther 2013;33:252-256

DOI: $10.1159 / 000342191$
Received: May 21, 2012

Accepted after revision: July 25, 2012

Published online: February 21, 2013

\title{
Fetal Cervical Teratoma: What Is the Role of Fetal MRI in Predicting Pulmonary Hypoplasia?
}

\author{
Katherine Wolfe ${ }^{a}$ David Lewis ${ }^{c}$ David Witte ${ }^{b}$ Beth Kline-Fath ${ }^{b}$ \\ Foong-Yen Lim ${ }^{b}$ Ronald Jaekle ${ }^{a}$ Mounira Hablib Judith Hostiuck ${ }^{a}$ \\ Naira Baregamian ${ }^{b}$ Sundeep Keswani ${ }^{b}$ Timothy Crombleholme ${ }^{d}$ \\ a University of Cincinnati College of Medicine and ${ }^{b}$ Cincinnati Children's Hospital Medical Center, Cincinnati, Ohio, \\ 'University of South Alabama, Mobile, Alabama, Ala., and ' University of Colorado School of Medicine, \\ Aurora, Colo., USA
}

\section{Key Words}

Cervical teratoma $\cdot \mathrm{MRI} \cdot$ Prenatal diagnosis $\cdot$ Pulmonary hypoplasia

\begin{abstract}
Objective: To determine whether total fetal lung volumes estimated by MRI could predict lethal pulmonary hypoplasia in a cohort of fetuses with cervical teratomas. Methods: We performed a retrospective cohort study of fetal cervical teratomas from January 1, 2005, through April 1, 2012. The primary outcome was the ability of total lung volumes measured by MRI to predict neonatal mortality specifically due to pulmonary hypoplasia. Measured lung volumes were compared to previously reported normal values. The percent of observed-to-expected lung volume and the percent predicted lung volume were calculated. The positive and negative predictive values were calculated for each variable. Results: Fetal MRI-derived total lung volumes 1 standard deviation below the median for gestational age had a positive predictive value of $100 \%$ in predicting lethal pulmonary hypoplasia. Conversely, total lung volumes above this level were uniformly associated with pulmonary survival $(100 \%$ negative predictive value). Additionally, percent predicted
\end{abstract}

lung volume $\leq 75.7$ and observed-to-expected lung volume $\leq 68.3$ were associated with lethal pulmonary hypoplasia. Conclusion: In this small cohort, MRI-estimated lung volumes were helpful in predicting the presence of pulmonary hypoplasia complicating fetal cervical teratoma.

Copyright $\odot 2013$ S. Karger AG, Basel

\section{Introduction}

Cervical teratoma, a germ cell tumor containing ectoderm, mesoderm and endoderm, is a rare fetal condition that can complicate the pregnancy, delivery and postnatal course. Cervical teratomas that are large in size can cause hyperextension of the neck in addition to potentially obstructing the airway or impeding fetal swallowing. As a result, polyhydramnios frequently occurs and increases the incidence of preterm labor and delivery. Hydrops and stillbirth have also been reported in association with cervical teratomas. A vaginal delivery is usu-

The content is solely the responsibility of the authors and does not necessarily represent the official views of the NIH.

\section{KARGER}

E-Mail karger@karger.com

www.karger.com/fdt (c) 2013 S. Karger AG, Basel

$1015-3837 / 13 / 0334-0252 \$ 38.00 / 0$
Timothy M. Crombleholme, MD, Colorado Fetal Care Center

Children's Hospital Colorado, Division of Pediatric General Thoracic and Fetal Surgery, University of Colorado School of Medicine

Anschutz Medical Campus, 13123 East 16th Ave, Aurora, CO 80045 (USA)

E-Mail timothy.crombleholme@ childrenscolorado.org 
ally not feasible due to the size of the teratoma and the hyperextension of the fetal head and neck.

The most common cause of death in neonates with a cervical teratoma is the inability to secure an airway after delivery. One of the most successful applications of the ex utero intrapartum treatment (EXIT) strategy has been in cases of giant cervical teratomas to allow time on placental support to secure the airway prior to delivery, reducing the risk of hypoxia and death [1-4]. In some cases, despite the success of the EXIT strategy in securing an airway, neonatal mortality can occur due to pulmonary hypoplasia associated with cervical teratoma. Pulmonary hypoplasia is an underappreciated complication of cervical teratoma, which has been reported in up to $30 \%$ of neonates born with a giant anterior cervical teratoma [5]. This may occur secondary to the neck mass severely hyperextending the fetal neck, pulling the carina up to or above the thoracic inlet, wedging the lungs into the apices of the chest cavity and impeding normal lung development [5].

The prognostic value of estimating fetal lung volume in fetuses with cervical teratomas is unknown. In fetuses with other etiologies of pulmonary hypoplasia, such as congenital diaphragmatic hernia, the fetal lung volume estimated on MRI is helpful, although institution specific, in predicting survival and the need for extracorporeal membrane oxygenation. Neonatal survival and short-term outcomes can be predicted based on comparisons with established normal ranges for lung volumes [3, 6-13]. If fetal lung volume in the setting of giant cervical teratomas can identify fetuses at risk for lethal pulmonary hypoplasia, we could more accurately counsel our patients on the survival of their fetus. In addition, identifying fetuses at high risk for lethal pulmonary hypoplasia would allow consideration for open fetal surgery to resect the mass, thereby allowing resolution of neck hyperextension and subsequent pulmonary development. In the absence of fetal surgery, low lung volumes would identify fetuses at high risk of mortality due to pulmonary hypoplasia despite the EXITto-airway strategy. The purpose of this study was to determine whether fetal lung volumes estimated by MRI in a cohort of fetuses with cervical teratomas could identify those at risk of lethal pulmonary hypoplasia.

\section{Materials and Methods}

Institutional review board approval was obtained from Cincinnati Children's Hospital Medical Center for this retrospective chart review. A retrospective cohort study was conducted on all patients diagnosed and evaluated for fetal cervical teratoma that were delivered at our center from January 1, 2005, through April 1, 2012. The Fetal Care Center of Cincinnati electronic database was searched for patients with a referral diagnosis or working diagnosis of either a cervical teratoma or a neck mass, or any patient who had an EXIT-to-airway procedure performed. The authors then reviewed the charts for eligible patients. Patients were included only if the prenatal diagnosis was cervical teratoma and the delivery was managed at our center. Demographic data, ultrasound findings, administration of betamethasone, obstetrical outcomes including polyhydramnios (AFI $>24 \mathrm{~cm}$ ) and hydrops (abnormal accumulation of fluid in at least two compartments), operative findings, neonatal survival were recorded. Neonatal autopsies were reviewed for cause of mortality. The director of Pathology and Laboratory Medicine (D.P.W.) reviewed the lung histology of each neonate that died. The pathologist was blinded to the fetal MRI lung volumes and diagnosed pulmonary hypoplasia qualitatively if the lung histology showed incomplete development for gestational age. Similarly, all fetal MRI images were reviewed by a single radiologist blinded to the outcome in these cases. Total lung volumes were calculated from a series of images on the fetal MRI. The series selected for calculation of total lung volumes were those that sequentially imaged the entire fetal lung in coronal plane without significant fetal motion artifact. The images were transferred to a Vitrea workstation on which the boundaries of the lungs were defined for calculation of a total lung volume. Lung volumes measured by fetal MRI imaging were compared to gestational age-matched normative values [14].

The primary outcome measure was the ability of fetal lung volumes measured by MRI to predict neonatal mortality specifically due to pulmonary hypoplasia prior to hospital discharge. Secondary variables included neonatal death from any cause, stillbirth and the method of obtaining an airway at the time of delivery. One mother had two fetal MRIs performed during her pregnancy, and in this case, the latter of the two studies was used in this study and lung volume analysis was not performed on the first MRI.

\section{Statistical Analysis}

Dichotomous variables were created for lung volumes below the median, 1 standard deviation or more below the median and 2 standard deviations or more below the median. Continuous variables were created for the percent of observed-to-expected lung volume and the percent predicted lung volume. The percent of observed-to-expected lung volume was calculated by dividing the measured volume on MRI by the expected mean volume and multiplying by 100 . The percent predicted was calculated by dividing the measured lung volume by the product of 0.0033 and gestational age to the square root of 2.86 as previously reported by Rypens et al. [14]. The positive and negative predictive values were calculated for each dichotomous variable for predicting lethal neonatal pulmonary hypoplasia. Statistical analyses were performed using Stata (Stata Statistical Software: Release 10. College Station, Tex., USA; StataCorp LP) using the MannWhitney U test for continuous variables and Fisher's exact test for categorical variables. Statistical significance was set at $\mathrm{p}<$ 0.05 . 
Table 1. Fetal findings on neonates with lethal pulmonary hypoplasia compared to pulmonary survivors

\begin{tabular}{lccc}
\hline & $\begin{array}{l}\text { Lethal pulmonary } \\
\text { hypoplasia }(\mathrm{n}=3)\end{array}$ & $\begin{array}{l}\text { Pulmonary survivor } \\
(\mathrm{n}=9)\end{array}$ & p value \\
\hline GA, weeks at evaluation & $30.1(23.6-32.9)$ & $30.1(23.7-34.7)$ & 0.64 \\
GA, weeks at delivery & $32(24.3-33)$ & $33.3(30.6-36.7)$ & 0.31 \\
Hydrops & $3(100)$ & $2(22.2)$ & 0.02 \\
Polyhydramnios & $3(100)$ & $8(88.9)$ & 0.75 \\
\hline Lung volumes $_{\text {Percent observed-to-expected }}$ & & $98.6(77.5-161.5)$ & 0.01 \\
Percent predicted $_{\text {Below median }}^{1}$ & $46.5(43.1-68.3)$ & $93.4(86.9-173.8)$ & 0.01 \\
1 SD below median & 1 & $5(55.6)$ & 0.25 \\
L SD below median $^{1}$ & $3(100)$ & $0(0)$ & 0.005 \\
\hline
\end{tabular}

Continuous variables are expressed as median (interquartile range) and compared using the Mann-Whitney U test. Categorical variables are expressed as number (\%) and compared using Fisher's exact test. GA = Gestational age.

${ }^{1}$ Measured lung volume compared to median and standard deviation (SD) of previously reported normal values [14].

\section{Results}

We reviewed the charts of 27 patients referred for a fetal neck mass. Thirteen fetuses were prenatally diagnosed with a cervical teratoma. Fourteen fetuses with fetal neck masses were excluded due to diagnosis other than cervical teratoma (lymphangioma $n=7$, hemangioma $n=2$, epignathus $\mathrm{n}=2$, intracranial teratoma $\mathrm{n}=1$, goiter $\mathrm{n}=1$, vascular malformation $\mathrm{n}=1$ ). One patient had a maternal medical contraindication to delivery via EXIT-to-airway and was managed at her home institution and therefore was excluded. Twelve patients were included in this study. Average gestational age at the time of imaging was $29^{3 / 7}$ weeks (range $23^{4 / 7}-34^{5 / 7}$ weeks). The average gestational age at delivery was $32^{3 / 7}$ weeks (range $24^{2 / 7}-36^{5 / 7}$ weeks). All women received a course of betamethasone for fetal lung maturity prior to delivery. No stillbirth occurred. Polyhydramnios occurred in 11 of 12 pregnancies. Hydrops occurred in 5/12 fetuses. EXIT-to-airway strategy was used for 11/12 patients and an airway successfully secured in all cases: 7 of 12 via orotracheal intubation and 5 with retrograde intubation via tracheotomy and repair. Neonatal death occurred in 5 of 12 fetuses: 1 case of massive hemorrhage during resection of the cervical teratoma secondary to venous hypertension and capillary leak syndrome on day of life (DOL) 9, 1 case of laryngotracheomalacia and tracheal collapse with attempted tracheotomy on DOL 30, and 3 cases of pulmonary hypoplasia (DOL 1-2).

Three neonatal deaths were attributed to pulmonary hypoplasia as documented in the discharge summary and autopsy report. One neonate died on DOL 1 from respiratory failure secondary to pulmonary hemorrhage and pulmonary hypoplasia. The second neonate died on DOL 2 from acute respiratory distress syndrome and disseminated intravascular coagulopathy. The third neonate died at 22 min of life despite cardiopulmonary resuscitation; the cause of death on autopsy was immaturity and pulmonary hypoplasia. These 3 neonates had pulmonary hypoplasia confirmed on histology. In contrast, the 2 neonates that died from other complications had normal pulmonary development on histology.

In this cohort, the prevalence of lethal pulmonary hypoplasia was $25 \%$. Table 1 compares the fetal findings on neonates with lethal pulmonary hypoplasia compared to pulmonary survivors. Those with lethal pulmonary hypoplasia had similar gestational ages at evaluation and delivery, and a similar rate of polyhydramnios compared to pulmonary survivors. The fetuses with lethal pulmonary hypoplasia were more likely to develop hydrops. The median percent predicted and observed-to-expected lung volumes were significantly greater in the pulmonary survivor group. The 3 neonates with lethal pulmonary hypoplasia all had fetal lung volumes more than 1 standard deviation below the median and statistically significantly differed from pulmonary survivors, all of whom had lung volumes within or above 1 standard deviation of the median ( $\mathrm{p}=0.005$, fig. 1 ). Fetal MRI-derived total lung volumes 1 standard deviation below the median for gestational age had a positive predictive value of $100 \%$ in predicting lethal pulmonary hypoplasia. Conversely, to- 


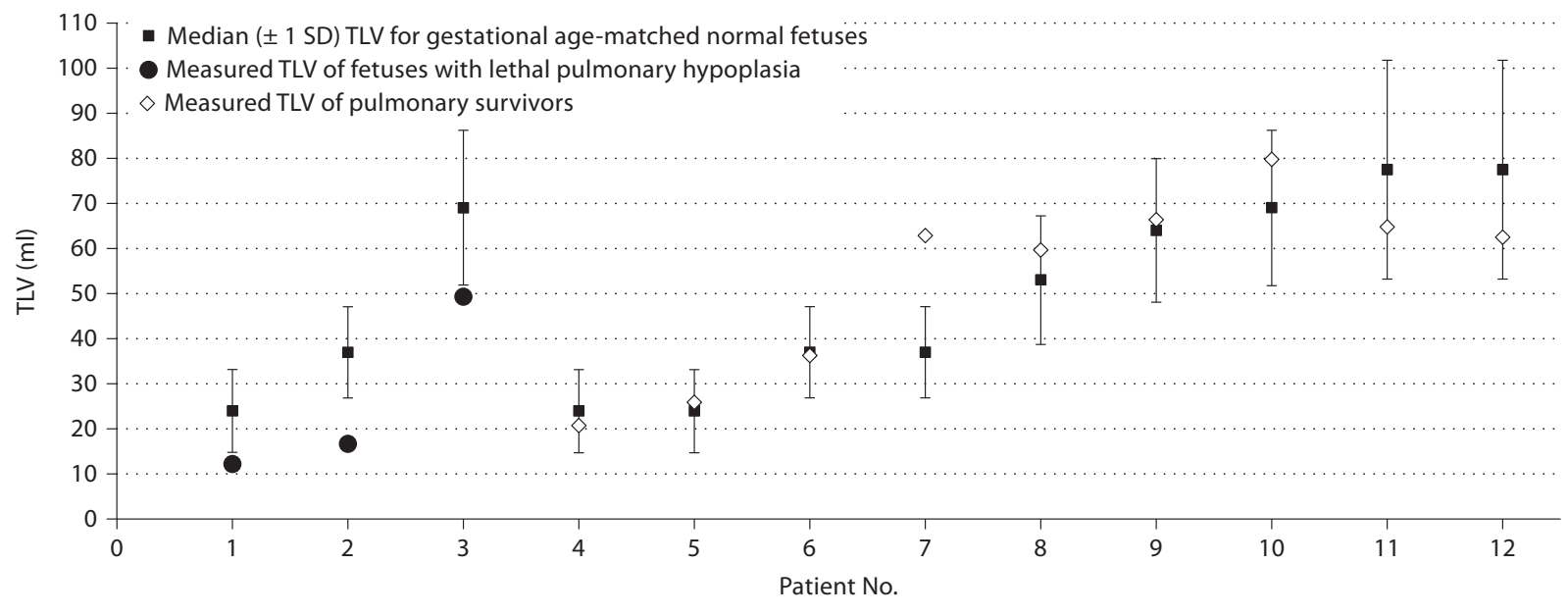

Fig. 1. Total lung volume (TLV) measured by MRI of 12 fetuses with anterior cervical teratomas compared to that of gestational age-matched normal fetuses (median $\pm 1 \mathrm{SD}$ ).

tal lung volumes above this level were uniformly associated with pulmonary survival $(100 \%$ negative predictive value). The 3 fetuses with lethal pulmonary hypoplasia had percent predicted lung volumes $\leq 75.7$ and percent observed-to-expected lung volumes $\leq 68.3$ while pulmonary survivors had greater lung volumes.

\section{Discussion}

Lethal pulmonary hypoplasia occurs in neonates with anterior cervical teratomas, which may be a consequence of hyperextension of the neck pulling the carina and the distal trachea up to the neck and the lungs into the thoracic apices. The presence of pulmonary hypoplasia can be predicted on prenatal MRI. In this small cohort of patients prenatally diagnosed with an anterior cervical teratoma, the prevalence of lethal pulmonary hypoplasia was $25 \%$. We found that fetal lung volumes 1 standard deviation or more below the median for gestational age had a high positive and negative predictive value in the setting of anterior cervical teratomas, and this threshold value was statistically significant when comparing neonates with lethal pulmonary hypoplasia to pulmonary survivors.

The limitation of this study is the small sample size, which makes it difficult to draw definitive conclusions. There were only 3 neonates with lethal pulmonary hypoplasia in this cohort of 12 fetuses with anterior cervical teratomas. Moreover, large anterior cervical teratoma is a rare condition. There are only a few larger series of prenatally diagnosed anterior cervical teratomas, none of which report predictors of pulmonary hypoplasia. The fetal MRIs in this cohort were performed during the evaluation of the neck mass, and not specifically for estimating total lung volumes. The gestational age at the time of MRI varied from 23.6 to 34.7 weeks. From previously reported studies, we know that lung growth is not linear, with accelerated lung growth occurring later in gestation. The optimal gestational age for MRI-estimated total lung volumes in the setting of a cervical teratoma is unknown. Furthermore, estimated lung volumes may be institution specific and we did not test the reproducibility of estimated fetal lung volumes in this study. Ideally, a prospective study would be done to evaluate the utility of using the cut-off points for percent predicted or observed-toexpected lung volume that were associated with lethal pulmonary hypoplasia in this cohort. Despite these limitations, obtaining total lung volumes in prenatally diagnosed cervical teratoma may prove useful in counseling patients.

Families of fetuses diagnosed with a fetal anterior cervical teratoma should be told that the neonate is at risk for lethal pulmonary hypoplasia. Families may decline the EXIT-to-airway procedure given the potential maternal risks and the implications for her future pregnancies when the neonatal prognosis is poor due to suspected pulmonary hypoplasia. On the other hand, open fetal re- 
section may be an option as success has been reported in 1 hydropic previable fetus with an anterior cervical teratoma [15]. Further studies to validate the proposed predictors of lethal pulmonary hypoplasia may allow clinicians to use fetal MRI estimates of total lung volume to guide decision-making in regard to fetal intervention in cases of cervical teratoma.

\section{Acknowledgment}

The project described was supported by the National Center for Research Resources and the National Center for Advancing Translational Sciences, National Institutes of Health, through Grant 8 UL1 TR000077-04.

\section{References}

1 Stevens GH, Schoot BC, Smets MJ, et al: The ex utero intrapartum treatment (EXIT) procedure in fetal neck masses: a case report and review of the literature. Eur J Obstet Gynecol Reprod Biol 2002;100:246-250.

-2 Tonni G, De Felice C, Centini G, Ginanneschi C: Cervical and oral teratoma in the fetus: a systematic review of etiology, pathology, diagnosis, treatment and prognosis. Arch Gynecol Obstet 2010;282:355-361.

-3 Liechty KW, Crombleholme TM, Flake AW, et al: Intrapartum airway management for giant fetal neck masses: the EXIT (ex utero intrapartum treatment) procedure. Am J Obstet Gynecol 1997;177:870-874.

4 Bianchi DW, Crombleholme TM, D'Alton ME, Malone FD: Cervical teratoma; in Fetology: Diagnosis and Management of the Fetal Patient, ed 2.. New York, McGraw-Hill, 2010, pp 751-757.

5 Liechty KW, Hedrick HL, Hubbard AM, et al: Severe pulmonary hypoplasia associated with giant cervical teratomas. J Pediatr Surg 2006;41:230-233.
6 Jani J, Cannie M, Sonigo P, et al: Value of prenatal magnetic resonance imaging in the prediction of postnatal outcome in fetuses with diaphragmatic hernia. Ultrasound $\mathrm{Ob}-$ stet Gynecol 2008;32:793-799.

7 Bonfils M, Emeriaud G, Durand C, et al: Fetal lung volume in congenital diaphragmatic hernia. Arch Dis Child Fetal Neonatal Ed 2006;91:F363-F364.

8 Zaretsky M, Ramus R, McIntire D, Magee K, Twickler DM: MRI calculation of lung volumes to predict outcome in fetuses with genitourinary abnormalities. AJR Am J Roentgenol 2005; 185:1328-1334.

-9 Gorincour G, Bouvenot J, Mourot MG, et al: Prenatal prognosis of congenital diaphragmatic hernia using magnetic resonance imaging measurement of fetal lung volume. Ultrasound Obstet Gynecol 2005;26:738-744.

10 Barnewolt CE, Kunisaki SM, Fauza DO, Nemes LP, Estroff JA, Jennings RW: Percent predicted lung volumes as measured on fetal magnetic resonance imaging: a useful biometric parameter for risk stratification in congenital diaphragmatic hernia. J Pediatr Surg 2007;42:193-197.
11 Terui K, Omoto A, Osada H, et al: Prediction of postnatal outcomes in congenital diaphragmatic hernia using MRI signal intensity of the fetal lung. J Perinatol 2011;31:269273.

12 Matsushita M, Ishii K, Tamura M, et al: Perinatal magnetic resonance fetal lung volumetry and fetal lung-to-liver signal intensity ratio for predicting short outcome in isolated congenital diaphragmatic hernia and cystic adenomatoid malformation of the lung. J Obstet Gynaecol Res 2008;34:162-167.

13 Kilian AK, Busing KA, Schuetz EM, Schaible T, Neff KW: Fetal MR lung volumetry in congenital diaphragmatic hernia $(\mathrm{CDH})$ : prediction of clinical outcome and the need for extracorporeal membrane oxygenation (ECMO). Klin Pädiatr 2009;221:295-301.

14 Rypens F, Metens T, Rocourt N, et al: Fetal lung volume: estimation at MR imaging-initial results. Radiology 2001;219:236-241.

15 Hirose S, Sydorak RM, Tsao K, et al: Spectrum of intrapartum management strategies for giant fetal cervical teratoma. J Pediatr Surg 2003;38:446-450; discussion 450. 